\title{
Machine Learning Based Correction Model in PMSM Power Loss Estimation for More-Electric Aircraft Applications
}

\author{
Yuan Gao \\ Power Electronics, Machines and \\ Control Group \\ University of Nottingham \\ Nottingham, UK \\ yuan.gao@nottingham.ac.uk \\ Serhiy Bozhko \\ Power Electronics, Machines and \\ Control Group \\ University of Nottingham \\ Nottingham, UK \\ serhiy.bozhko@nottingham.ac.uk
}

\author{
Tao Yang \\ Power Electronics, Machines and \\ Control Group \\ University of Nottingham \\ Nottingham, UK \\ tao.yang@nottingham.ac.uk \\ Pat Wheeler \\ Power Electronics, Machines and \\ Control Group \\ University of Nottingham \\ Nottingham, UK \\ pat.wheeler@,nottingham.ac.uk
}

\author{
Xin Wang \\ Institute for Aerospace Technology \\ University of Nottingham \\ Nottingham, UK \\ xin.wang2@,nottingham.ac.uk
}

\begin{abstract}
This study utilizes the machine learning (ML) technique to estimate the power loss of surface-mounted Permanent Magnet Synchronous Motor (PMSM) for MoreElectric Aircraft (MEA). Existing approaches do not consider ML methods in power loss calculation and only depend on empirical correction factors. The proposed ML aided model is proved to be more precise. Matching the analytical loss estimation with finite-element analysis (FEA) is the main research goal which includes two aspects: iron loss and permanent magnet (PM) loss. They are both based on conventional formulae but this study analyzes the limitation of these equations and the ML correction model can provide dedicated factors for the analytical motor model to make sure that the loss estimation is accurate in the whole motor design space. Average correction factor (ACF) approach is regarded as the comparison method to verify the excellent performance of the proposed ML model.
\end{abstract}

Keywords-Machine learning (ML), artificial neural network (ANN), PMSM, power loss correction, more-electric aircraft (MEA)

\section{INTRODUCTION}

With the continual development of magnet material, permanent magnet (PM) machines have been widely applied in electrical vehicles, fans, drives, and compressors due to their high efficiencies [1-6]. And PM machines are commonly selected for electromechanical actuation in MEA concepts and applications [4,7]. However, the problem of designing an optimal PM machine is challenging because of the multiphysics nature of its operation. Several computational models can account for the multi-physics operation, including the closed-form analytical model, lumped parameter model, finite-element analysis (FEA) model, combined finite-element and analytical model, etc. Among that, FEA models are the most time consuming to solve but they are most accurate. Therefore, time-step FEA can also be an effective tool to verify loss calculation based on simpler analytical loss models since it is impractical to verify all loss predictions with experiments [2].

On the other hand, the design of a motor usually begins with geometries when the motor topology has been confirmed [5-7]. For permanent magnet synchronous motor (PMSM), its weight can be given mathematically with high accuracy by using the selected geometry parameters and materials, as long as the Mean Length per Turn (MLT) in a coil is properly estimated. In contrast, power loss (PL) estimation is more difficult to do as the flux field is coupled with many factors and flux density varies much among back-iron, tooth, PM and rotor. In that case, the analytical approach is prone to lose the accuracy since traditionally only the flux density of typical points is utilized instead of considering the whole iron area.

In [2], a classical estimation model considering both hysteresis and eddy-current losses was proposed; however, the correction factors obtained by theoretically derived formulas are substantial and yield inaccurate results due to missing the domain wall motion. Thus, this classical model was improved by adding an excess eddy current loss term in [3] which can be useful but needs many iterations for the motor initial design.

This study proposes a new method for loss estimation of machine stator and PM realized by using machine learning (ML) method to bridge the gap between analytical and FEA models. It utilizes simple conventional equations and databased ML training thus no need for substantial derivations. More importantly, ML based correction model can give dedicated factors to ensure the accuracy of loss estimation in the whole motor design space. Noting that the proposed MLbased approach should not be restricted to iron and PM loss in the motor of the MEA actuator. Based on FEM/experimental data collection, this approach can be also applied to the holistic loss estimation of the motor even the whole actuation system.

\section{FUNDAMENTALS OF LOSS ESTIMATION}

\section{A. Iron Loss}

Under the no-load condition, iron losses contribute to the largest part of the total motor loss. To calculate it in the soft ferromagnetic material, the modified Steinmetz equation is used with two different terms, hysteresis and eddy current loss. The specific iron loss ratio $W_{i r}(\mathrm{~W} / \mathrm{kg})$ for a certain material is given as [5]:

$$
W_{i r}(f, \hat{B})=K_{h} f^{\alpha} \hat{B}^{\beta}+K_{e}\left(K_{s f} f \hat{B}\right)^{2}
$$

where $\hat{B}$ is the peak amplitude and $f$ is the motor electrical frequency, $K_{s f}$ is the stacking factor of the lamination sheets. 
$K_{h}, \alpha, \beta$ and $K_{e}$ are Steinmetz coefficients determined by fitting the loss data from manufactures for specific materials.

The assumption of using Eq. (1) is that the stator flux density is a sinusoidal wave. However, in fact, the flux densities in the stator tooth tip and in the back-iron parts linking the stator base are far from sinusoidal, even approximate linear relations in the time domain. Moreover, as discussed, analytical models traditionally use the densities of typical points to estimate stator losses for the sake of efficient computation which generates another difference between analytical and FEA model, the magnitude, that means non-uniformed flux distribution on the stator. Therefore, iron-loss estimation of using Eq. (1) at one or several typical points is prone to have large errors especially for different geometry and winding parameters. That underlies the interest of new analytical approaches.

The iron material used in this study is M235-35A steel whose Steinmetz coefficients can be found in Table I.

TABLE I. PARAMETERS OF M135-35A StEEL

\begin{tabular}{|c|c|c|c|}
\hline Density $\left(\mathbf{k g} / \boldsymbol{m}^{\mathbf{3}}\right)$ & 7600 & $\begin{array}{c}\text { Lamination } \\
\text { thickness }(\boldsymbol{m m})\end{array}$ & 0.35 \\
\hline $\boldsymbol{K}_{\boldsymbol{h}}$ & 0.0081294 & $\boldsymbol{\alpha}$ & 1.208357 \\
\hline $\boldsymbol{K}_{\boldsymbol{e}}$ & 3.442366 & $\boldsymbol{\beta}$ & 1.78619 \\
\hline
\end{tabular}

\section{B. PM Loss}

Three significant parts of motor losses are usually considered in the PM exited synchronous machines: iron losses, winding losses, and eddy current losses in PM [8]. However, in some optimization-oriented motor design models, power losses of PM are usually not accounted for $[5,7]$. Two analytical arrangements for the surface-mounted PM were studied to estimate the eddy current loss: one is a simplified rectangle model, see Fig. 1(a); the other is using a plate of a sector of the circular ring which is depicted in Fig. 1(b).

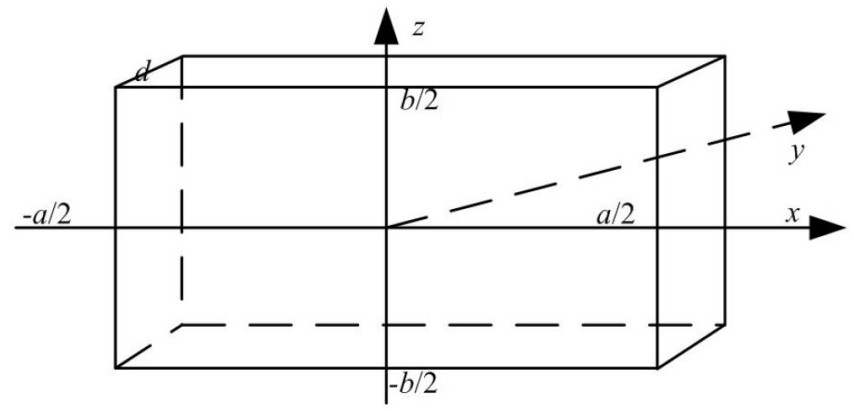

(a) Rectangular solid of PM [9]

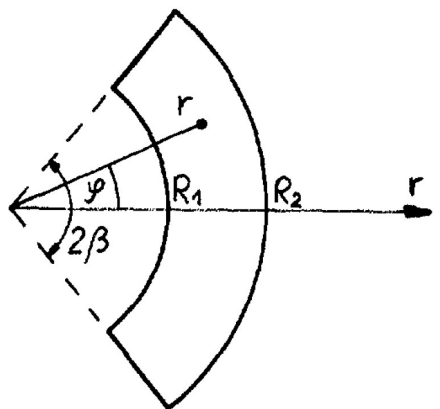

(b) Shape of a sector of circular ring [10]

Fig. 1 Two arrangements for surface-mounted PM.
In the former, $a$ denotes the width of a PM, $b$ is the axial length of PM or motor. Thus, the PM flux direction is mainly in the $y$-axis. Both Eq. (7) and Eq. (8) in [9] were tested in this study with current input but it appears that they are not feasible for a different motor topology because [9] only focuses on the interior permanent magnet machines. Thus, this study employs the second way for PM and Eq. (2) from [10] is utilized to predict PM losses:

$$
P_{P M}=\frac{1}{12}(2 \pi f)^{2} \hat{B}_{P M}^{2} b \sigma R_{2}^{4}\left(1-k_{c}^{2}\right)\left(1-k_{c}\right)^{2} \beta_{1}(1-
$$

where $\hat{B}_{P M}$ is the estimated peak flux density in PM, $\sigma$ is the conductivity of PM material, $\beta_{1}$ is the half of the circular angle of PM (rad). $R_{1}$ and $R_{2}$ are the internal and external radius of the PM circular ring, $c$ and $k_{c}$ are defined as

$$
\begin{gathered}
c=\sqrt{\frac{2\left(1-k_{c}^{2}\right)\left(1-k_{c}\right)^{2}}{\left(1-k_{c}^{2}\right)\left(1-8 k_{c}+k_{c}^{2}\right)-12 k_{c}^{2} \ln \left(k_{c}\right)}}, \\
k_{c}=\frac{R_{1}}{R_{2}} .
\end{gathered}
$$

Compared with the methods in [9], the prediction performance of this circular-ring method is more acceptable for the studied PMSMs; however, as will be shown in the case (Section IV), the relative errors of PM-loss predictions are still very big. With the help of the proposed ML correction model, the PM losses can be given very close to the FEA results in a certain motor design space. The following section will introduce the proposed ML model and its comparison method in detail.

\section{AVERAge CORRECTION FACTOR AND PROPOSED ML BASED MODEL}

Before talking about the proposed ML method for loss correction, this study introduces the average correction factors (ACFs) as a conventional method for contrastive analysis. The basic thinking of ACF is firstly using the boundary values of design variables (DVs) to provide correction factors for analytical loss estimation of PM, tooth, back-iron, and then validate these factors using more sample data in a motor optimization problem. ACF not only provides a comparison method to give correction factors but verifies the performance of its own average operation.

As will be shown in Section V, the ACF method is feasible when DVs are designed in the small ranges but will fail when we extend their ranges. Noting that the factors stay unchanged for all design points in a specific design space while ML based correction model can generate adaptive correction factors for different designs.

\section{A. Average Correction Factor}

The ACF operation process is depicted in Fig. 2. First, we assume three correction factors all equal 1 and there are $n$ DVs. Second, the first sample collection is exercised by only using up/down boundary values for each DV (this sampling scheme is named as "sweep2"), i.e. that we have $2^{n}$ design points to do the joint simulation (Math and FEA) and collect loss results in this round. After that, the initial three correction factors should be updated by a simple average operator using 2 groups of $2^{n}$ loss values: 


$$
k_{n e w_{-} 1}^{i t e m}=\frac{1}{2^{n}} \sum_{j=1}^{2^{n}} \frac{P L_{F E A_{-} j}^{i t e m}}{P L_{M A T_{-} j}^{i t e m}}
$$

where item means three studied motor parts: PM, yoke (stator back-iron), and stator tooth, $P L_{F E A_{-} j}^{i t e m}$ denotes the power loss of FEA at the $j$-th design point, $P L_{M A T_{-} j}^{\text {item }}$ is the power loss of math model at the same point. In the next step, replace initial correction factors with the corresponding $k_{n e w_{-}}$in the math model and do the second round of collection where evenly sample 4 values for each DV (sweep4, includes 2 boundary values). Further, use the same average operator to calculate the second new factors:

$$
k_{n e w_{-} 2}^{\text {item }}=\frac{1}{4^{n}} \sum_{j=1}^{4^{n}} \frac{P L_{F E A_{-} j}^{\text {item }}}{P L_{M A T_{-} j}^{\text {item }}}
$$

whose only difference with Eq. (5) is operating on $4^{n}$ loss values instead of $2^{n}$. If all three $k_{n e w_{2} 2}$ are close to 1 with small errors and the relative error of three-part loss $\left(E_{r}\right.$, Eq. (7)) is smaller than $3 \%$, validation is successful, otherwise, the validation fails.

$$
E_{r}=\frac{P L_{M A T}^{A l l}-P L_{F E A}^{A l l}}{P L_{F E A}^{A l l}}
$$

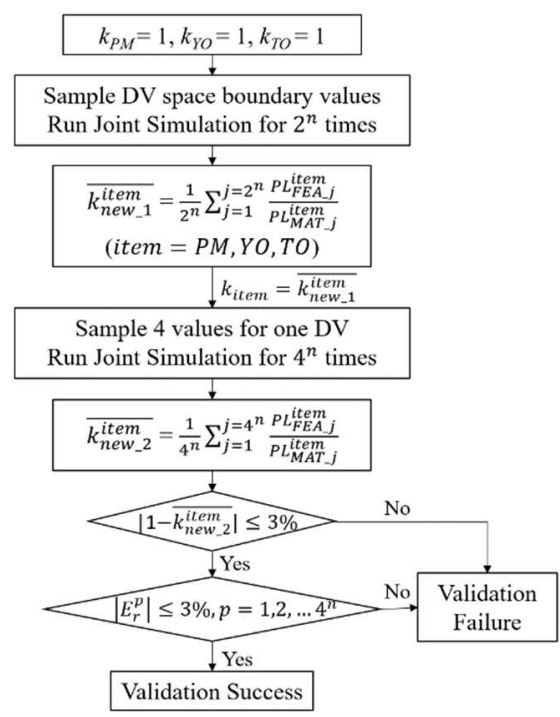

\section{Fig. 2 The methodology of Average Correction Factor}

This paper will give a 3-DV case in Section V using ACF and explain it in detail with different DV ranges.

\section{B. Proposed ML Based Correction Model}

This paper proposes a ML based correction model to give the correction factors $\left(k_{i t e m}\right)$ for motor loss estimation. This model is implemented by establishing a forward Artificial Neural Network (ANN) which is a common and powerful learning method in ML, deep learning and artificial intelligence areas [11-14]. ANN is based on a nonparametric regression model which is a technique for supervised learning. The user does not need to specify the relationship between the predictors (input) and responses (output) since ANN can learn them automatically by using only several training parameters (i.e. weights and bias). ANN fundamentals can be found in Section IV. B of [13], no more details.

As shown in Fig. 3, the analytical formulation for the desired correction model is constructed by using a 3-layer
ANN. In particular, this network represents the following relation:

$$
y=F(x) \Leftrightarrow\left(k_{P M}, k_{Y O}, k_{T O}\right)=F_{1}\left(D V_{1}, D V_{2} \cdots D V_{n}\right) .
$$

This relation clearly shows the network mapping from DVs to three $k_{\text {item }}$ which serve as a fast, accurate, and flexible correction model for loss estimation. The deployment of this ANN tool includes normalization functions at the input $(i)$ and de-normalization function at the output $(o)$. The numbers of hidden layers and neurons in each layer are pre-defined by trial-and-error. This study only uses one hidden layer and twenty neurons for the sake of convenience but, as will be discussed in Section $\mathrm{V}$, it can give excellent prediction performance after the ANN training.

ANN should be trained by sample data which is obtained by the Matlab-MotorCAD joint simulation. This study suggests using the second sample collection in the last subsection (sweep4, $4^{n}$ samples) where the relative errors $\left(E_{r}^{p}\right)$ of the proposed model should be much smaller than ACF.

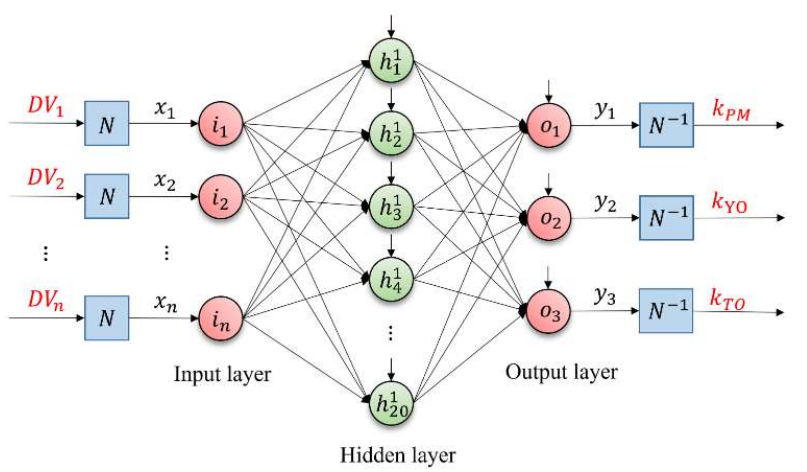

Fig. 3 Proposed ANN based correction model. For simplicity, weights and bias terms are omitted from the figure.

\section{CASE STUDY}

In this section, a PMSM case (12 slots 10 poles) is given with 3 DVs using both ACF and the proposed ANN tool to estimate the loss of PM, yoke, and tooth. The selected DVs are motor axial length $(b)$, PM height $\left(d_{m}\right)$, and RMS current (I). The analytical motor sizing model is from [5] which comprehensively includes the geometrical and winding model, electromagnetic model, and thermal model, but this study only utilized the first two models to focus on the PL estimation.

To compare the performance of two methods, two groups of data ranges are given to $3 \mathrm{DVs}$, as shown in Table II. Other parameters of the motor stay unchanged in this study (summarized in Table III). The following subsections will give the PL results using both ACF and ANN.

TABLE II. DATA RANGES OF 3 DESIGN VARIABLES

\begin{tabular}{|c|c|c|}
\hline & Small & Large \\
\hline$b(\mathrm{~mm})$ & {$[60,70]$} & {$[50,70]$} \\
\hline$d_{m}(\mathrm{~mm})$ & {$[4.5,5]$} & {$[4.5,5.5]$} \\
\hline$I(A)$ & {$[8.49,9.19]$} & {$[7.87,9.9]$} \\
\hline
\end{tabular}

TABLE III. PARAMETERS OF MOTOR DESIGN CASE 


\begin{tabular}{|c|c|c|c|}
\hline Rated speed (rpm) & 1500 & $\begin{array}{c}\text { Electrical } \\
\text { frequency (Hz) }\end{array}$ & 125 \\
\hline $\begin{array}{c}\text { Peak phase voltage } \\
(\mathbf{V})\end{array}$ & 77.23 & $\begin{array}{c}\text { No. of turns per } \\
\text { phase }\end{array}$ & 120 \\
\hline $\begin{array}{c}\text { Wire diameter } \\
(\mathbf{m m})\end{array}$ & 0.682 & $\begin{array}{c}\text { Depth of tooth } \\
\text { base (mm) }\end{array}$ & 19.67 \\
\hline $\begin{array}{c}\text { Depth of tooth tip } \\
\text { (mm) }\end{array}$ & 1.26 & $\begin{array}{c}\text { Tooth tang angle } \\
\text { (Deg) }\end{array}$ & 38.38 \\
\hline Air gap (mm) & 1.3 & $\begin{array}{c}\text { Rotor radius } \\
\text { (mm) }\end{array}$ & 28.6 \\
\hline Yoke Depth (mm) & 6.88 & PM fraction & $88.19 \%$ \\
\hline Tooth tip fraction & $78.83 \%$ & Tooth Fraction & $60.33 \%$ \\
\hline
\end{tabular}

\section{A. Small Ranges}

In this small range case, every DV varies in a small data space thus the motor loss will not have a large variation. Based on that, ACF can be a suitable way for loss correction since it utilizes the mean value to reflect the overall variation of PL. Following the afore-mentioned ACF process, this study exercised two rounds of joint simulation, sweep2, and sweep4. After that, the relative error results $\left(E_{r}^{p}\right)$ can be obtained by Eq. (7) which should be compared with zero.

Correction factors in this small-range case can be found in the second row of Table IV. All $3 k_{n e w_{-} 2}$ values are close to 1 with their errors smaller than $3 \%$. Thus, we can continue to sum the PL values of PM, yoke, tooth, and then get $E_{r}^{p}$ results, depicted in Fig. 4(a). It shows that 64 error values are all in [$3 \%, 3 \%$ ] which means the validation of ACF is successful. Further, to validate the method using more data, this study exercised the $3^{\text {rd }}$ Round of joint simulation, sweep5. The resulting data are shown in Fig. 4(b) which also verifies the fact that ACF method can get acceptable results in the small ranges $\left(\left|E_{r}\right| \leq 3 \%\right)$

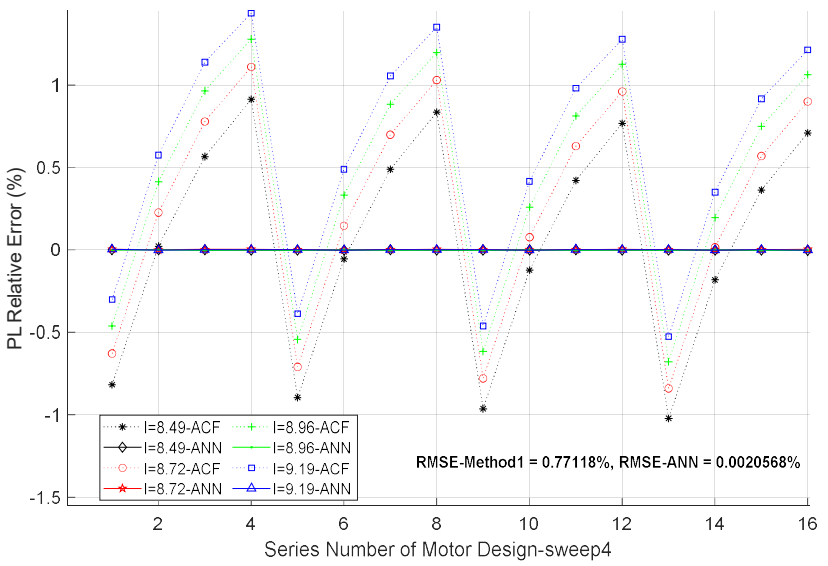

(a) sweep4 results of $E_{r}$

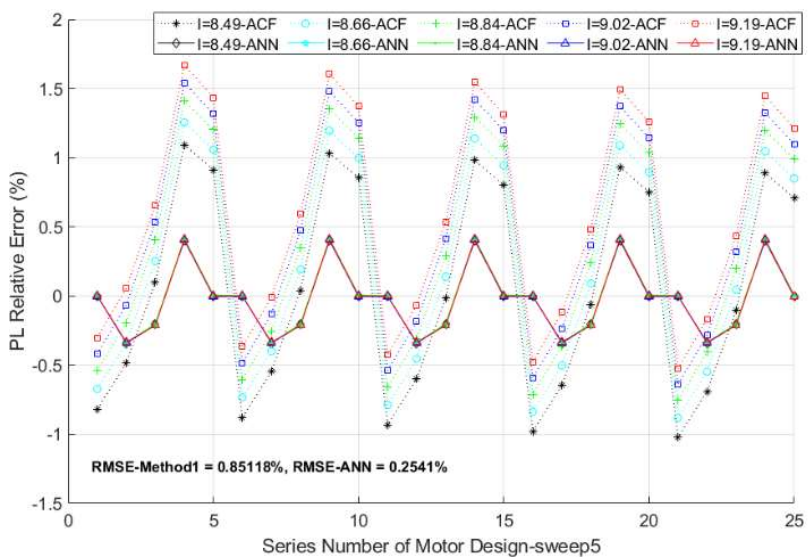

(b) sweep5 results of $E_{r}$

\section{Fig. 4. Relative PL error results in small ranges}

It is noted that, to show the 4D-point results (3 DVs plus PL error) in one surface, the $x$-axis in the figures of this Section denotes the series number of combinations of two DVs, $b$ and $d_{m}$, thus there are 16 combinations in sweep4 and 25 for sweep5. And we have each line for a combination set with regards to the other DV, $I$.

For the proposed ANN correction model, 64 samples (from sweep4) are regarded as the training data. As the sample number is not much, the training process only costs a few seconds in Matlab on a standard PC. After training, the network tool serves as a fast and convenient correction function to give the right factors $\left(k_{\text {item }}\right)$ to every design point of math model. The correction performance of this tool is depicted in Fig. 4 where the left figure demonstrates the excellent ANN training performance and the right figure shows the big gain of the ANN tool compared with ACF.

\section{B. Large Ranges}

Similarly, this study defined the large ranges for $3 \mathrm{DV}$ s to check the feasibility of ACF and ANN by doing three rounds of joint simulations: sweep2, sweep4, and sweep5. As shown in the third row of Table IV, since the value of $k_{\text {new_2 }}$ is not close to 1 , ACF method validation fails in the large ranges. But this study continues to compute $E_{r}$ results of ACF to be compared with the proposed ANN tool. Comparison results are discussed as follows.

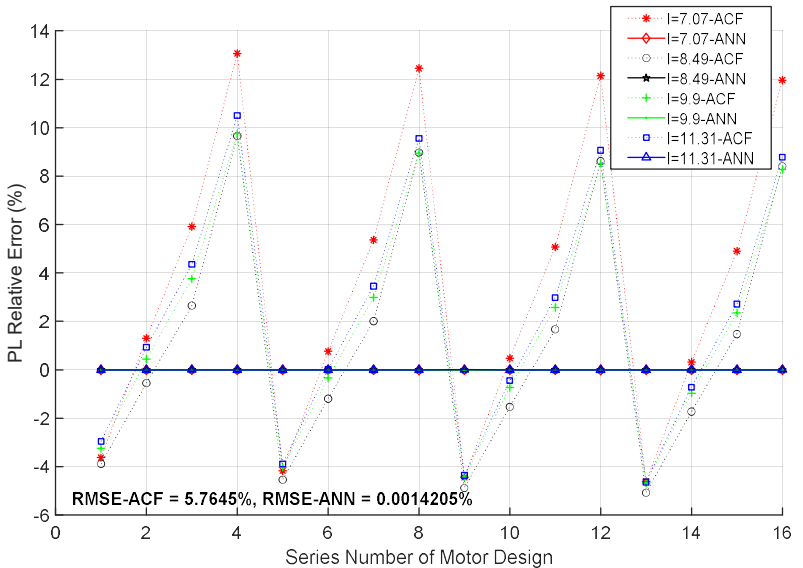

(a) sweep4 results of $E_{r}$

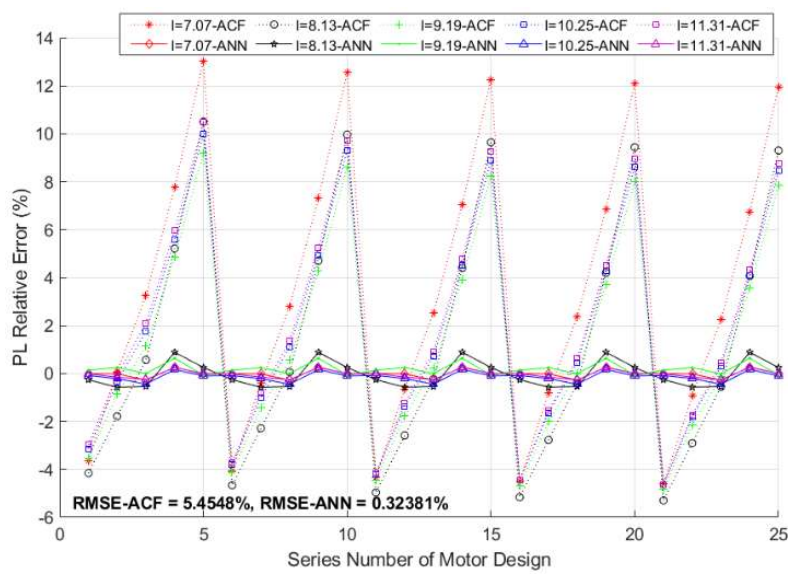

(b) sweep5 results of $E_{r}$

Fig. 5. Relative PL error results in large ranges

Fig. 5 shows the comparison results of large ranges. Most of $E_{r}$ values in sweep4 and sweep5 using ACF have exceeded 
$[-3 \%, 3 \%]$ but all absolute $E_{r}$ values using ANN are smaller than $1 \%$. Results of sweep4 demonstrate excellent ANN training performance. Even test the ANN tool using sweep5 data (noting that three-fifth of them are not ANN training data), the RMSE value of 125 points is only $0.324 \%$. In contrast, the RMSE of the ACF method is around 5.45\%.

TABLE IV. ACF RESULTS

\begin{tabular}{|c|c|c|c|c|c|c|}
\hline & $\boldsymbol{k}_{\text {new_1 }}^{P \boldsymbol{M}}$ & $\boldsymbol{k}_{\text {new_1 }}^{\boldsymbol{Y O}}$ & $\boldsymbol{k}_{\text {new_1 }}^{T O}$ & $\boldsymbol{k}_{\text {new_2 }}^{P M}$ & $\boldsymbol{k}_{\text {new_2 }}^{\boldsymbol{P O}}$ & $\boldsymbol{k}_{\text {new_2 }}^{T \boldsymbol{O}}$ \\
\hline $\begin{array}{c}\text { Small } \\
\text { ranges }\end{array}$ & 0.2522 & 1.2133 & 1.2973 & 1.0276 & 1.0077 & 0.9979 \\
\hline $\begin{array}{c}\text { Large } \\
\text { ranges }\end{array}$ & 0.2223 & 1.2072 & 1.2865 & $\mathbf{1 . 1 0 2 7}$ & 1.0022 & 0.9921 \\
\hline
\end{tabular}

\section{CONCLUSION}

This paper proposes a simple, convenient and dedicate correction model for motor loss estimation based on training an artificial neural network. The fundamentals of loss estimation used in this work are given and, average correction factor method is introduced as a comparison method. Lastly, a motor of $12 \mathrm{~S} 10 \mathrm{P}$ is discussed and the results of both methods are depicted to demonstrate the excellent performance of the proposed ANN approach.

\section{ACKNOWLEDGMENT}

This project has received funding from the Clean Sky 2 Joint Undertaking under the European Union's Horizon 2020 research and innovation programme under grant agreement Number 807081.

\section{REFERENCES}

[1] G. R. Slemon, "High-efficiency drives using permanent-magnet motors," in Proc. Int. Conf. Industrial Electronics, Control and Instrumentation, vol. 2, Maui, Hawaii, 1993, pp. 725-730.

[2] Chunting Mi, G. R. Slemon and R. Bonert, "Modeling of iron losses of permanent-magnet synchronous motors," in IEEE Transactions on Industry Applications, vol. 39, no. 3, pp. 734-742, May-June 2003.

[3] W. Roshen, "Iron Loss Model for Permanent-Magnet Synchronous Motors," in IEEE Transactions on Magnetics, vol. 43, no. 8, pp. 34283434, Aug. 2007.

[4] P. Giangrande, V. Madonna, G. Sala, A. Kladas, C. Gerada and M. Galea, "Design and Testing of PMSM for Aerospace EMA
Applications," in IECON 2018 - 44th Annual Conference of the IEEE Industrial Electronics Society, 2018.

[5] B. Cheong, P. Giangrande, X. Zhang, M. Galea, P. Zanchetta and P. Wheeler, "Fast and Accurate Model for Optimization-based Design of Fractional-Slot Surface PM Machines," 2019 22nd International Conference on Electrical Machines and Systems (ICEMS), Harbin, China, 2019, pp. 1-6.

[6] G. Bramerdorfer, J. A. Tapia, J. J. Pyrhonen and A. Cavagnino, "Modern Electrical Machine Design Optimization: Techniques, Trends, and Best Practices," IEEE Transactions on Industrial Electronics, vol. 65, pp. 7672-7684, 102018.

[7] B. Cheong, P. Giangrande, M. Galea, P. Zanchetta and P. Wheeler, "Integrated motor drive design for weight optimization," 2017 IEEE Energy Conversion Congress and Exposition (ECCE), Cincinnati, $\mathrm{OH}$, 2017, pp. 816-823.

[8] Schmidt, Erich, Manfred Kaltenbacher, and Anton Wolfschluckner. "Eddy current losses in permanent magnets of surface mounted permanent magnet synchronous machines-Analytical calculation and high order finite element analyses." $e$ \& $i$ Elektrotechnik und Informationstechnik 134.2 (2017): 148-155.

[9] M. Cheng and S. Zhu, "Calculation of PM Eddy Current Loss in IPM Machine Under PWM VSI Supply With Combined 2-D FE and Analytical Method," in IEEE Transactions on Magnetics, vol. 53, no. 1, pp. 1-12, Jan. 2017, Art no. 6300112.

[10] R. Sikora, J. Purczynski, W. Lipinski and M. Gramz, "Use of variational methods to the eddy currents calculation in thin conducting plates," in IEEE Transactions on Magnetics, vol. 14, no. 5, pp. 383385, September 1978.

[11] Z. Xu, Y. Gao, X. Wang, X. Tao and Q. Xu, "Surrogate Thermal Model for Power Electronic Modules using Artificial Neural Network," IECON 2019 - 45th Annual Conference of the IEEE Industrial Electronics Society, Lisbon, Portugal, 2019, pp. 3160-3165.

[12] Y. Gao, T. Yang, S. Bozhko, P. Wheeler and T. Dragičević, "Filter Design and Optimization of Electro-Mechanical Actuation Systems Using Search and Surrogate Algorithms for More-Electric Aircraft Applications," in IEEE Transactions on Transportation Electrification, doi: 10.1109/TTE.2020.3019729.

[13] T. Dragičević, P. Wheeler and F. Blaabjerg, "Artificial Intelligence Aided Automated Design for Reliability of Power Electronic Systems," in IEEE Transactions on Power Electronics, vol. 34, no. 8, pp. 71617171, Aug. 2019.

[14] S. Wang, T. Dragicevic, Y. Gao, S. K. Chaudhary and R. Teodorescu, "Machine Learning based Operating Region Extension of Modular Multilevel Converters under Unbalanced Grid Faults," in IEEE Transactions on Industrial Electronics, doi: 10.1109/TIE.2020.2982109. 